\title{
Organ Procurement Processes in the Operating Room: The Effects of an Educational Session on Levels of Confidence and Understanding in Operating Room Registered Nurses and Surgical Technologists
}

Follow this and additional works at: https://www.journal.acorn.org.au/jpn

Part of the Perioperative, Operating Room and Surgical Nursing Commons, and the Surgery Commons (c) (i)

This work is licensed under a Creative Commons Attribution 4.0 License.

\section{Recommended Citation}

Ross, Ann; Reilly, Janet; Halla, Emily; and Anderson, Kathryn (2020) "Organ Procurement Processes in the Operating Room: The Effects of an Educational Session on Levels of Confidence and Understanding in Operating Room Registered Nurses and Surgical Technologists," Journal of Perioperative Nursing: Vol. 33 : Iss. 2 , Article 2.

Available at: https://doi.org/10.26550/2209-1092.1072

https://www.journal.acorn.org.au/jpn/vol33/iss2/2

This Article is brought to you for free and open access by Journal of Perioperative Nursing. It has been accepted for inclusion in Journal of Perioperative Nursing by an authorized editor of Journal of Perioperative Nursing. 


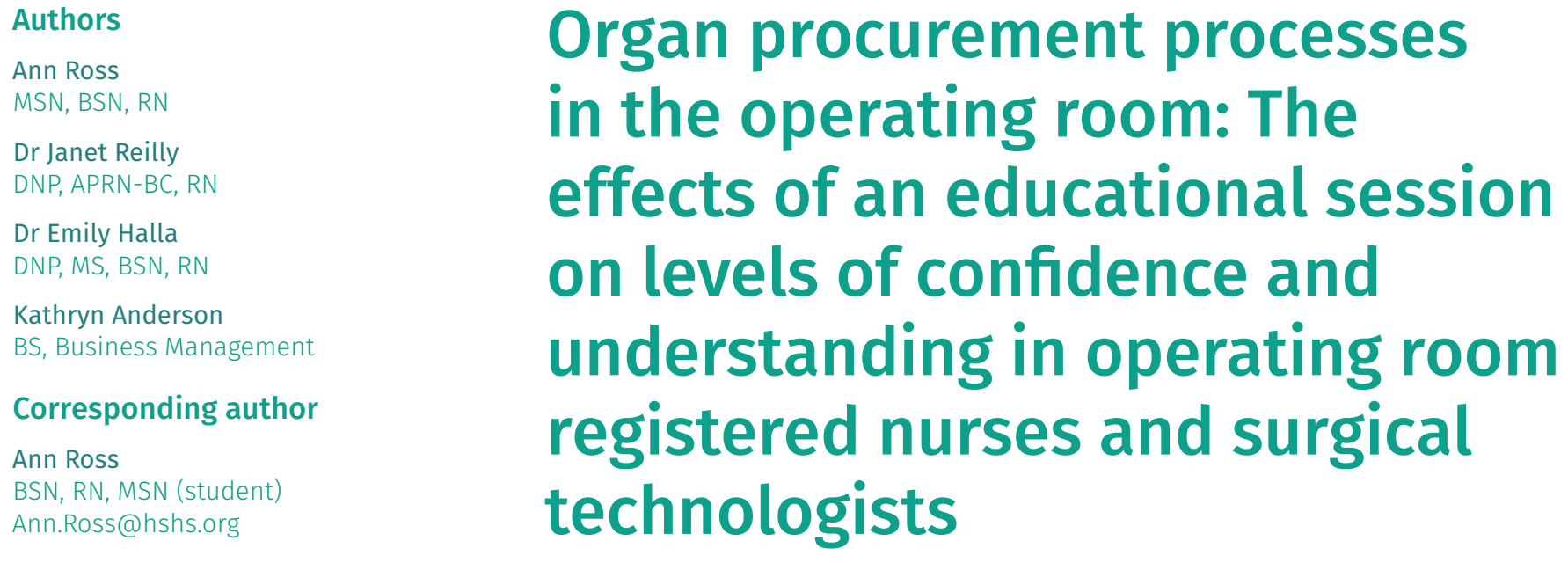

Abstract

Objectives: This project aimed to determine the effects an online educational course about organ procurement procedures (OPPs) in the operating room (OR) had on the levels of confidence and understanding among OR registered nurses (RNs) and surgical technologists (STs).

Sample and setting: Data was collected from OR RNs and STs in the United States (US) at a Level II trauma centre with 19 operating rooms that completes eight to ten OPPs per year. A graduate nursing leadership student in this hospital created a Microsoft PowerPoint educational course about OPPs as her capstone project.

Methods and variables: The course was developed and offered through the online learning management system the hospital uses. A retrospective pretestposttest survey was designed and evaluated the effects of the course on participants' levels of confidence and understanding.

Results: The overall levels of understanding and confidence about OPPS improved in the RNs and STs, comparing aggregate scores.

Implications for perioperative nursing: An educational course can enhance understanding of and confidence in OPPs for OR RNs and STs. Increased confidence and understanding can advance the successful outcomes of the OPPs. Ongoing education is needed to reinforce confidence and understanding in rarely done surgical procedures like OPPS.

\section{Introduction}

Lack of organs for donation and transplantation is a global issue. For example, in 2018, over 113000 people in the United States (US) awaited organ transplantation, with 20 people dying each day while waiting. Similar need is evident in Australia. Global comparison of organ donation rates is measured in donors per million of population (DPMP) which considers changes in population over time; Australia's DPMP is 20.8 compared to the US at $31.72^{2}$. The lack of available organs for transplant is complex. Only three in one thousand deaths occur in a way that allows the deceased persons' organs to be eligible for donation and subsequent transplant, either through donation after brainstem death (DBD) or donation after circulatory (cardiac) death (DCD) 1,3,4. The limited availability of eligible donated organs compared to the number of people awaiting transplantation prioritises 
the need for successful outcomes in organ procurement and transplant procedures.

In the US, organ transplant procedures can only be completed by an authorised organ procurement organisation (OPO) ${ }^{5}$. However, the organ procurement procedure (OPP) can occur in any hospital in the US that has an operating room (OR), ventilator and the staff with the ability to manage a patient requiring ventilation. The OR registered nurses (RNs) and surgical technologists (STs) that participate play an important role in the success of OPPs in the US. RNs in the US are licensed and have completed a two- to four-year nursing program at a technical or university college. The RNs function in the role of the circulator in the $\mathrm{OR}$, ensuring the team works together for the patient's best interest. While not a role in Australia, STs in the US are trained through a specialised program at local technical colleges. Their focus is on handling the instruments in the operating room.

While the literature addresses education that RNs receive about patient care prior to the OPP, it is limited in regard to education that RNs and STs receive about patient care during the OPP. This education is predominantly hospital-based and provided either as in-service training sessions taught by the OPO organ donor coordinators or offered during OR employee orientation, neither of which include the RN or ST responsibilities during the $\mathrm{OPP}^{6}$. Lack of, or insufficient, organ and tissue donation procedure education is a global issue and the need for more specialised education for health professionals closely involved in OPPs has been identified by international studies $7,8,9,10,11$. In both Australia and the US, dedicated teams to complete OPPs are not always available in smaller hospitals and those in less urban areas?
Therefore, OR RNs and STs who may be less familiar with OPPs participate in these procedures, possibly feeling vulnerable and more uncertain about their roles and responsibilities?. Education about OPPs can alleviate some of their uncertainty and result in successful functioning and job satisfaction $^{12}$.

\section{Objectives}

This project was developed by a nursing graduate student in a leadership and management program. The focus was to determine the effects of a self-directed education session about OPPs for OR RNs and STs on their level of understanding and confidence in the processes, using a retrospective pretest-posttest design.

\section{Sample and setting}

The project setting was a non-profit, 517-bed hospital in the midwestern US, which is part of a health care system with fifteen hospitals and numerous clinics across two states. Fifty-nine RNs and STs were employed in the hospital OR and invited to participate in the project. The OR RNs and STs verbalised to the nurse educator concerns about their lack of comfort providing care during OPPs. This hospital completes eight to ten OPPs per year (almost equal numbers of DBD and DCD) and works with a regional OPO, located about 300 kilometres away.

\section{Methods and variables}

The project was deemed Institutional Review Board (IRB) exempt for the graduate student's university and the hospital. An education session was developed by the nursing graduate student to help address OR RNs and STs' concerns. Throughout its development, the education session was edited with feedback from the RN and ST managers, regional OPO staff and two hospital nurse educators. The session was offered to OR RNs and STs through the hospital's online learning management system. All 59 RNs and STs employed received an automated email when the session was available to be completed with periodic email reminders to encourage completion prior to the due date. Participants were able to log on and complete the self-directed education session individually on any hospital computer during work time. The content in the session was identified in the literature ${ }^{6,13,14}$ and included an overview of OPP, perioperative responsibilities for OR RNs and STS, information on the criteria for DBD and DCD, and post-mortem care. Content was presented in a format similar to a PowerPoint presentation. The education session also included a video showing a meeting between organ donor and recipient family members, which is voluntary in the US. The education session took 30 minutes to complete and was available for one month. Responses were de-identified except by role (RN or ST). After completing the session, the participants were directed to complete an anonymous online survey which identified their levels of confidence and understanding before and after the OPP education session.

The survey used a retrospective pretest-posttest design, which allowed each participant to rate his or her perceptions before and after the training from the same frame of reference. This design can remove response shift bias ${ }^{15}$. This form of evaluation was also used because individuals 'may not know what they do not know' nor be able to accurately reflect and selfreport their level of knowledge and confidence prior to participation in the educational session ${ }^{16}$. Before answering survey questions, informed consent was obtained from participants after they read 
a summary of the project title, investigators, purpose, procedure, benefits, risk, safeguards, and IRB approval (see additional files).

The survey consisted of demographic questions (Q1-5) and quantitative questions regarding participants' understanding and confidence in OPPs (Q6-11) that were answered using a five-point Likert-type scale ('strongly agree', 'agree', 'neutral', 'disagree', 'strongly disagree'). Two qualitative, open-ended questions were also asked (Q12-13). Deidentified results of the survey questions were analysed in the aggregate.

\section{Results}

The OPP session was completed by 41 RNs and STs employed in the hospital (69\%). Eighteen of these RNs $(n=11)$ and STs $(n=7)$ also consented and completed the survey. The majority of survey participants $(72 \%, n=13)$ had participated in less than five OPPs and had varied surgical experience. Six participants (33\%) had more than 15 years of experience in their current OR position, while seven participants (39\%) had worked less than five years. More than half of the participants $(56 \%, n=10)$ indicated they had some form of formal education or training about OPPs prior to completing this online education session, mostly through in-service trainings (Q5).

Aggregate survey responses to Q6-11 noted increased confidence and understanding related to OPPs after the education session compared to before the session (see Figure 1 below). For example, the number of responses rating confidence in the ability to explain two types of OPPs (DBD, DCD) improved after completing the education session (78\%; $n=14)$, compared to before the education session ( $50 \% ; n=9$ ). Similarly, confidence to perform OPPs changed from 83\% ( $n=15)$ who 'agreed'

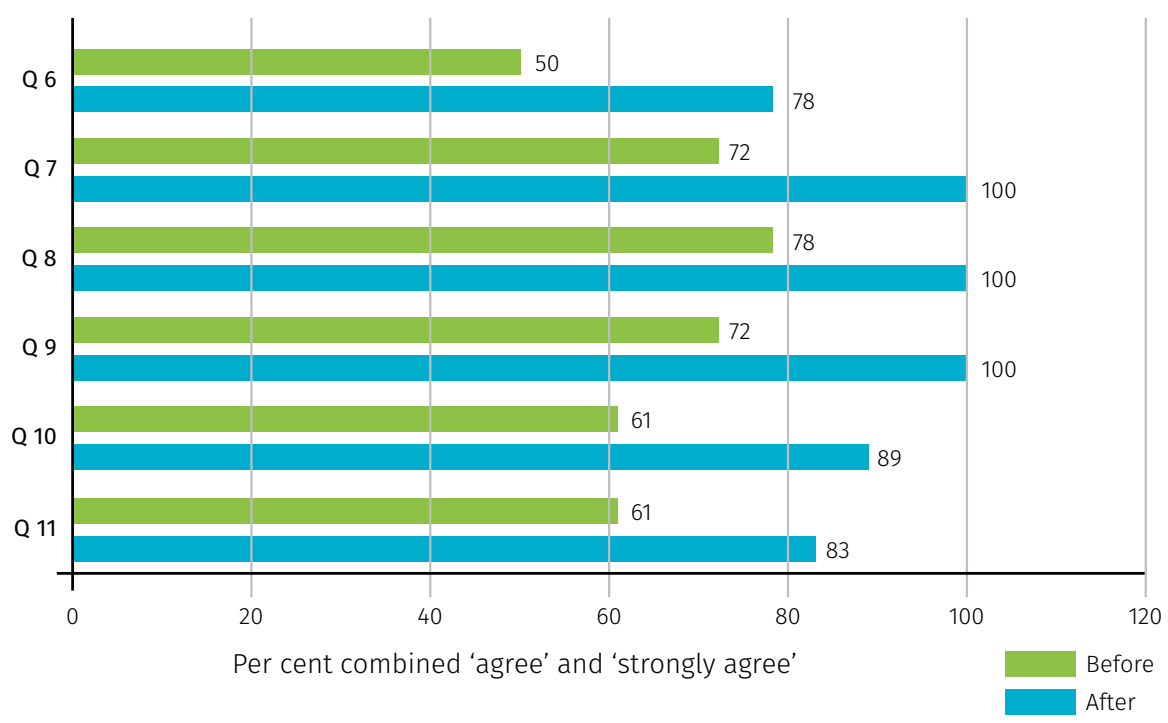

Figure 1: Comparison of survey responses before and after the education session

or 'strongly agreed' (collectively hereafter called as 'agreed') after session completion, compared to $62 \%$ $(n=11)$ before completing the session. Unanimous agreement was reported by participants after completing the education session in three areas. All eighteen participants (100\%) 'agreed' they were able to differentiate the two types of OPPs, and they understood their responsibilities before and during OPPS.

\section{Survey responses}

Participants identified what they learned that would help them the next time they participated in an OPP. These were information about set up for each of the rooms $(n=3)$, charting or paperwork to be completed $(n=3)$, differences between DBD and DCD $(n=2)$, and roles $(n=2)$. For example, participants' responses included learning whether anaesthesia was needed for the OPP and knowing that family members were able to be in the OR. Information that RNs and STs stated should be included in future educational sessions involved the processes before patients come into the OR $(n=2)$, charting and forms $(n=1)$, what happens after OPPs, time frames for transplantation $(n=1)$ and OPP in-service for new OR RN and STs $(n=1)$. One participant echoed the purpose of this project by saying an educational session can be helpful to new employees or people who do OPPs infrequently to 'know what it's like to be in a transplant procedure just so you are more prepared'.

\section{Implications for perioperative nursing}

There are important implications from this project, although they are not generalisable nor statistically significant due to the small sample size. After the education session, every survey question related to participants' levels of understanding and confidence in OPPs improved. Participants were also able to identify things they could apply in future OPPS. Despite participants having previous education and surgical experience, the need for ongoing specialised education for those participating in OPPs was found, as supported by the literature? 


\section{Knowledge translation}

Ongoing education, in an easily accessible and convenient format, needs to be provided for RNs and STS in each hospital in the US and RNS in Australia where OPPs may take place ${ }^{6}$. A standardised presentation of the OPP information from the education session in this project could be customised for use in other hospitals. Similar education could increase participants' understanding and confidence in other infrequent surgical procedures.

\section{Funding}

No monies were received, and the authors have no financial interests.

\section{References}

1. Health Resources \& Services Administration (HRSA). Organ donation statistics [Internet]. Rockville, USA: HRSA; 2018. Available from: www.organdonor.gov/statistics-stories/ statistics.html.

2. ShareLife. Australian organ donation performance-leading practice and global comparison [Internet]. Sydney: ShareLife; 2018. Available from: www.sharelife.org.au/ australian-organ-donation-comparison.
3. National Health Service, UK. Blood and Transplant. Donation after brainstem death [Internet]. London: NHS; Available from: odt. nhs.uk/deceased-donation/best-practiceguidance/donation-after-brainstem-death /

4. National Health Service, UK (NHS). Blood and Transplant. Donation after circulatory death [Internet]. London: NHS; Available from: odt.nhs.uk/deceased-donation/ best-practice-guidance/donation-aftercirculatory-death/.

5. Health Resources and Services Administration (HRSA). Organ Transplant and Procurement Network policies [Internet]. Rockville, USA: HRSA; 2019. Available from: optn.transplant.hrsa.gov/ media/1200/optn_policies.pdf.

6. Smith Z, Leslie G, Wynaden D. Experiential learning not enough for organ procurement surgery: Implications for perioperative nursing education. Prog Transplant 2015;25(4):339-350

7. Smith Z, Woods D, Lea J, Usher K. Australian perioperative nurses' attitudes, levels of knowledge, education and support needs related to organ donation and procurement surgery: A national survey. JPN 2019;32(2):7-15.

8. Collins TJ. Organ and tissue donation: A survey of nurse's knowledge and educational needs in an adult ITU. Intensive Crit Care Nurs 2005;21(4):226-233.

9. Kim JR, Fisher MJ, Elliott D. Attitudes of intensive care nurses towards brain death and organ transplantation: Instrument development and testing. J Adv Nurs 2006;53(5):571-582
10. Flodén A, Forsberg A. A phenomenographic study of ICU-nurses' perceptions of and attitudes to organ donation and care of potential donors. Intensive Crit Care Nurs 2009;25(6):306-313.

11. Meyer K, Bjфrk IT, Eide H. Intensive care nurses' perceptions of their professional competence in the organ donor process: A national survey. J Adv Nurs. 2012;68(1):104-115.

12. Carter-Gentry D, McCurren C. Organ procurement from the perspective of perioperative nurses. AORN J 2004;80(3):417431.

13. Gao W, Plummer V, Williams A. Perioperative nurses' attitudes towards organ procurement: A systematic review. J Clin Nurs 2017;26(3/4):302-319.

14. Lyons P, Treasure E. The design and implementation of an organ donation education workshop for perioperative nurses. ACORN J 2014;27(1):28-29.

15. Chang R, Little TD. Innovations for evaluation research: Multiform protocols, visual analog scaling, and the retrospective pretest-posttest design. Eval Health Prof 2018;4(2):246-269.

16. Brook J, Akin B, Lloyd M, Bhattarai J, McDonald T. The use of prospective versus retrospective pretests with child-welfare involved families. J Child Fam Stud 2016;25(9):2740-2752. 